PAPER

\title{
Effective speech suppression using a two-channel microphone array for privacy protection in face-to-face sales monitoring
}

\author{
Osamu Ichikawa*, Takashi Fukuda and Ryuki Tachibana \\ IBM Research, 5-6-52, Toyosu, Koto-ku, Tokyo, 135-8511 Japan \\ (Received 30 January 2015, Accepted for publication 5 August 2015)
}

\begin{abstract}
In the financial industry, face-to-face conversation is an essential for sales. Similar to call-center monitoring, there is a significant need to monitor the conversation for compliance checks. In certain business scenarios, there is a need to record an employee's speech while protecting the customers' confidentiality and privacy. In this paper, we propose a small-scale microphone array system specially designed to record only the agent's speech. For the suppression of the customer's speech, we used CSP-based post-filtering. However, using small number of microphones, it is difficult to suppress unwanted speech completely. Because post-filtering using correlations of the multiple channels often affected by the spatial aliasing between speakers. We introduced the weighted-CSP to attenuate susceptible bins to the interfering speaker. Also we introduced flooring after the post-filtering to mask residuals. This combination helps prevent the customer's speech to be transcribed.
\end{abstract}

Keywords: Microphone array, Post-filtering, Generalized cross-correlation, Cross-power spectrum phase, Beamformer

PACS number: 43.60.Fg [doi:10.1250/ast.36.507]

\section{INTRODUCTION}

Automatic speech recognition (ASR) is now being widely used by consumers, since most smartphones have voice assistance. Also in business, there are tremendous needs for speech-based solutions. Call-center monitoring is a good example. In most systems, the agent's speech and the customer's speech on the telephone line are recorded separately by a logger and also transcribed separately. This is because primary purpose of this solution is a compliance check, to detect agents saying something inappropriate. A secondary purpose is to detect unhappy customers who should be brought to a supervisor's attention.

In the financial industry, the face-to-face interaction with an agent at a teller-counter is a main sales channel. Similar to call-center monitoring, there is a need to monitor these conversations. Unfortunately, it is impractical to obtain customer's approval before recording the conversation, so we started this study based on our assumption that we could avoid seeking the customer's approval if the recording device does not record the customer's speech, but only the agent's speech.

The simplest method for this type of recording is to use a close-talk microphone positioned next to the agent's

*e-mail: ichikaw@jp.ibm.com mouth, but this can leave customers with a negative impression of the interaction. Also, it will still pick up small amount of the customer's speech. A directional pinmicrophone worn on the agent's clothing may not show a negative impression, but a pin-microphone will pick up the customer's speech considerably.

We explored beamformer technology to satisfy the requirements defined as,

1. The customer's speech must be blocked almost completely. It should not be transcribed by ASR, as well as being inaudible for human.

2. The agent's speech must be well recognized by ASR.

3. The microphone array needs to be small scale such as two-microphone.

In general, the more microphones installed, the higher performance the beamformer has. However, it may require special hardware. That could end up with unacceptable cost if they were deployed to all branch offices. Therefore, our assumption uses two microphones only, so that we can use inexpensive audio devices. Another benefit of the two microphone approach is that the computational cost can be reduced, so that the algorithm can be implemented as software in a personal computer in a branch office or in a cloud server.

This paper focuses on speech suppression and proposes an improved post-filtering that is designed to minimize the 


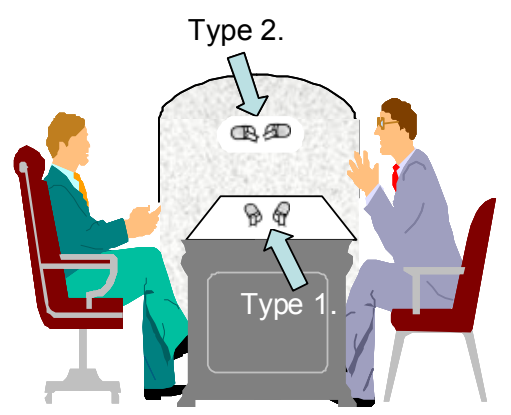

Fig. 1 Microphone setup for teller's counter.

interference from the customer's speech. Another claiming point of this paper is to place the post-filtering between spectral subtraction (SS) and flooring. In this way, the residual sound may become less audible by the flooring.

\section{PRIOR ARTS}

Many researches have been made for two-microphone approaches using a near-end microphone and a far-end microphone to a subject speaker.

One approach is binary-masking [1-3]. It performs a component selection per DFT bin for each frame. It uses inter-microphone level difference (ILD) or inter-microphone phase difference (IPD) as a clue to determine which speaker occupies the bin based on the sparseness assumption.

We have a specific geometry in this study shown in Fig. 1. Unidirectional microphones are suitable to observe the power difference. However, there is a problem that they do not work as a phased array in this configuration, because a sound wave coming from the back-side generates a flipped phase with a unidirectional microphone.

On the other hand, omnidirectional microphones can observe IPD correctly, although ILD is not available in this configuration. It should be noted that there is a restriction that the distance between microphones needs to be small so to secure IPD information. Many previous attempts used a narrow array with a few centimeters spacing or a lower sampling frequency such as $8 \mathrm{kHz}$ so to avoid spatial aliasing. As binary-masking approach is often combined with beamformer technologies [1,3], the limitation of the distance is an issue for the total performance. For our proposed system, larger distance is applied with upper bound frequency for the binary-masking.

As regards beamformers, various technologies have been studied in the past. A delay and sum beamformer focuses in a target direction by synchronizing the channels. A minimum variance beamformer [4] minimizes the ambient noise by maintaining constant gain in the target direction. For extra noise reduction, subtraction of estimated noise by a sub-beamformer was explored by the Griffiths-Jim beamformer [5] and Spatial Spectral Sub- traction [6]. For decomposition, the profile fitting method [7] uses multiple directional templates to work with directional and non-directional (diffusive) sound sources. Nonnegative Matrix Factorization (NMF) [8] was enhanced as multi-channel NMF [9].

These speech separation methods have various pros and cons in adaptation speed and timing, computational costs, processing delays, etc. However one shared aspect that none of them completely eliminate sounds from an antitarget direction, especially with small numbers of microphones. They often have small residual components from other directions than the target. Unfortunately, ASR is often more sensitive to such residual speech than humans, allowed recognition of some of these words, and so we need to completely suppress this residual component.

For noise suppression, post-filtering is often added. The basic form is Zelinski's post-filter [10] and it has been extended in many ways [11-13]. However, Zelinski's postfilter assumes noise is uncorrelated between channels. Therefore, it cannot efficiently suppress directional noise we are tackling. If it is used at larger microphone distance, it has substantial amount of residual noise, because it designs the filter at each frequency bin regardless to the spatial aliasing. On the other hand, Nagata used GCCPHAT as the metric of the correlation between channels in order to suppress directional noise [14]. Unlike Zelinski's post-filter, it designs a single filter for all the frequency bins. Therefore, it is essentially a gain control function. It can efficiently suppress directional noise in a noise-only segment, while it does very little when a subject speaker and directional noise are both active. However, it should be noted that it still keeps the suppression in short pauses when a subject speaker talks. This is unlike a simple segmentation approach based on DOA or power. Actually, it significantly contributes ASR accuracy.

In this paper, we used GCC-PHAT-based post-filter for the starting point of our improvement, because it better performed in our experiments than Zelinski's one.

In this paper, we call GCC-PHAT-based post-filter for CSP-based post-filter (CSP_PF), because Cross Spectrum Phase (CSP) [15] is equivalent to GCC-PHAT and our proposed post-filter uses weighted version of CSP. We call it for weighted-CSP-based post-filter (w-CSP_PF). It should be noted that weighted-CSP has been studied mainly in sound source localization area [16,17]. However, this paper investigated a new weighted-CSP for the postfiltering. For the sound source localization, a standard CSP was used.

In the situation we assume in this paper, an agent and a customer basically speak alternately. Mixed speech is rarely observed. Therefore, if we can completely suppress the output by post-filtering when the unwanted speaker solely speaks, most of our cases are solved. For mixed 
speech segments, an existing beamformer technology can help to attenuate unwanted speaker's voice. Even though it is not completely eliminated, the attenuated speech in a mixed speech segment is hard to perceive.

\section{PROPOSED SYSTEM}

\subsection{Overview}

As stated in Sect. 1, our objective is to block customer's speech almost completely while it retains agent's speech in the face-to-face configuration using small number of microphones such as two. In order to achieve this goal, we combined our new technology components and existing components listed below;

- New components

1. Weighted-CSP-based post-filter (w-CSP_PF).

2. Flooring placed after post-filter.

- Existing components

1. Minimum Variance beamformer.

2. DOA-based binary mask.

New component 1 is the main contributor. It significantly extends the suppression capability of CSP_PF. New component 2 was also effective to mask residual noise with a scaled stationary noise.

\subsection{Microphone Installation}

As shown in Fig. 1, the agent and customer are usually seated face-to-face across a counter. Our approach is to place two omnidirectional microphones between them on a line parallel to both participants. This is because the CSP index corresponding to the sound source direction (DOA) has a lower resolution along the array lines shown in Fig. 2, which makes it significantly easier to track the speakers' positions. Our system has a DOA tracking mechanism, but it also works well with the fixed DOA indices such as +7 and -7 , allowing for some postural shifts by the speakers [18].

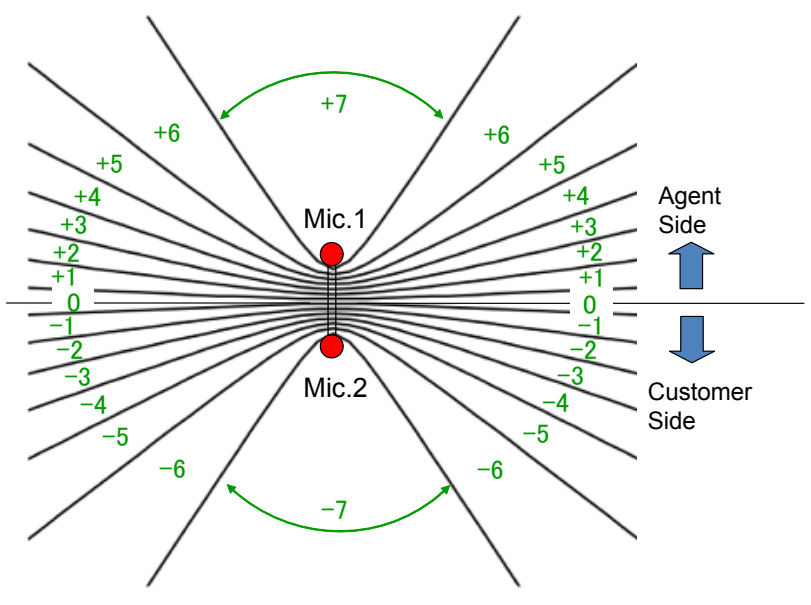

Fig. 2 Example of CSP index.

The placement of the microphone pair allows for some flexibility. As shown in Fig. 1, it can be placed on the counter or on the partitioning wall of a booth. Please note that the wall case (Type 2) still has a geometry requirement for pointing to the speakers.

When the sampling frequency is set to $22.05 \mathrm{kHz}$, the distance between the two microphones should theoretically be less than $1.5 \mathrm{~cm}$ so to avoid spatial aliasing. However, such a narrow array causes poor performance in noise reduction, especially for lower frequencies. Therefore we used a separation distance around $12 \mathrm{~cm}$ with some risk of spatial aliasing. The spatial aliasing occurs at lower frequencies when the two participants are widely separated, as in this situation. The CSP coefficient used in our postfiltering was affected by spatial aliasing, which will be discussed in Sect. 3.5.

\subsection{System Configuration}

Figure 3(a) shows the diagram of our proposed system 1. For the speech separation, a Minimum Variance

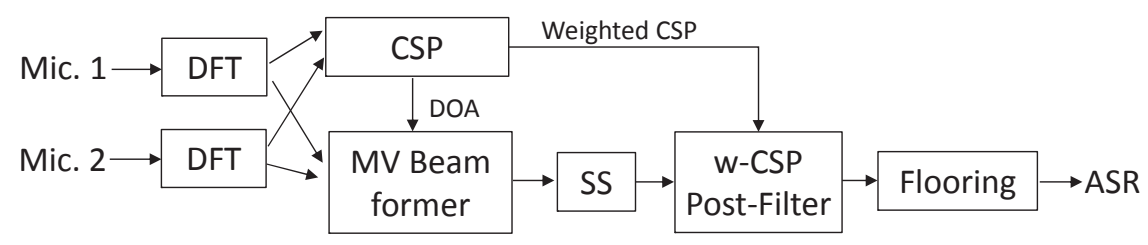

(a) Proposed system 1.

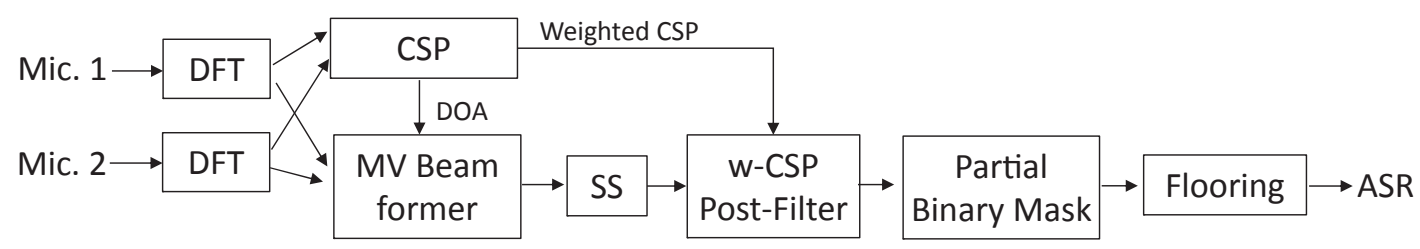

(b) Proposed system 2.

Fig. 3 Block diagram of proposed systems. 
BeamFormer (MVBF) is used. In a quiet environment, this should form a directional null for the interfering voice. In this study, the interfering speaker is the customer. The MVBF output is also processed with Spectral Subtraction (SS) to reduce the stationary ambient noises such as air conditioners or personal computers. In each frame, a CSP analysis is also done with the two channel inputs to find the peak index of the DOA for the MVBF beamformer. The CSP coefficient at that peak index is also used for the post filtering. It is extended to weighted-CSP in Sect. 3.5. In this paper, (weighted) CSP coefficient to the target direction is written as CSP-target. When the target speaker does not speak, CSP-target reaches zero to minimize the output. It should be noted that the flooring is placed not after SS but after the post-filtering. In general, an ASR decoder uses logarithmic features such as MFCC and is sensitive to near-zero fluctuations. To resist recognizing faint voices the output of the post filtering is floored by the scaled stationary noise [19].

The proposed system 1 can be combined with partialbinary-masking as shown in Fig. 3(b) proposed system 2. As we have larger distance between microphones than the standard binary-masking configuration, we introduced upper frequency limiting binary-masking, which will be discussed in Sect. 3.6.

\subsection{CSP Analysis and Post-filtering}

CSP analysis measures the normalized correlations between its two microphone inputs with an Inverse Discrete Fourier Transform (IDFT). Weighted-CSP is written as

$$
\varphi_{T}(i)=\operatorname{IDFT}\left[W_{T}(k) \cdot \frac{S_{1, T}(k) \cdot S_{2, T}(k)^{*}}{\left|S_{1, T}(k)\right| \cdot\left|S_{2, T}(k)\right|}\right],
$$

where $S_{m, T}$ is a complex spectrum in the $T$-th frame as observed with microphone $m$ and $*$ is the complex conjugate. The DFT bin number $k$ corresponds to the frequency. $W(k)$ is the weight for the bin $k$ and it is set to 1.0 for standard (non-weighted) CSP analysis. Our proposed system uses standard CSP analysis for DOA estimation and weighted-CSP for the post-filtering whose $W(k)$ will be defined in Sect. 3.5.

The CSP coefficient $\varphi_{T}(i)$ is a time-domain representation of the normalized correlation for the $i$-sample delay. For a stable representation, a moving average of the CSP coefficients is introduced as $\bar{\varphi}_{T}$ using several frames around $T$. The estimated DOA of the target speaker as $\hat{\imath}_{T}$ can be found within the region of the agent side, using

$$
\hat{\imath}_{T}=\underset{o<i<i_{\max }}{\operatorname{argmax}}\left(\bar{\varphi}_{T}(i)\right)
$$

The maximum index is given by

$$
i_{\max }=d \cdot f / c \text {. }
$$

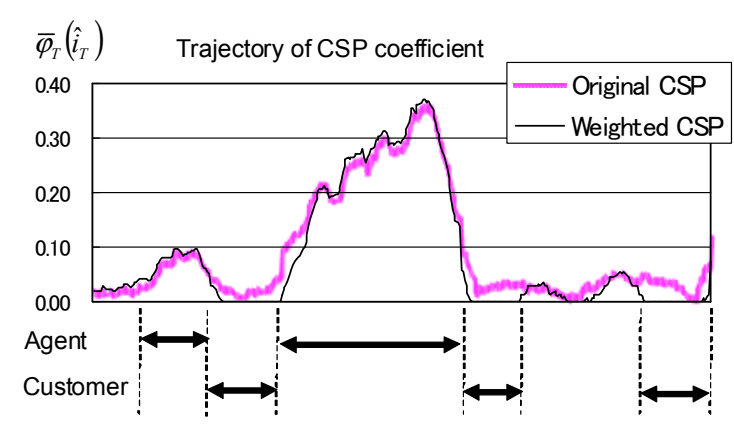

Fig. 4 Trajectory of CSP coefficient for the target direction (CSP-target).

Here, $c$ is the acoustic velocity, $d$ is the distance between microphones and $f$ is the sampling frequency.

This is the DOA to be passed to the MVBF. Note that $\hat{\imath}_{T}$ will be unchanged when the CSP peak is found on the customer side or when no one is speaking. For the postfiltering, the SS output $X_{T}$ is multiplied by the CSP-target as $\bar{\varphi}_{T}\left(\hat{\imath}_{T}\right)$.

$$
Y_{T}(k)=\left\{\max \left(\bar{\varphi}_{T}\left(\hat{\imath}_{T}\right), \quad 0.0\right)\right\} \cdot X_{T}(k) .
$$

Here, $X_{T}$ is the power spectrum of the SS output and $Y_{T}$ is the value after post-filtering. It is processed with the flooring with the stationary noise component $N$.

$$
Z_{T}(k)=\max \left(Y_{T}(k), \quad \beta \cdot N(k)\right) .
$$

Here, $\beta$ is the flooring coefficient. We set it to 0.2 in our experiments. $Z_{T}$ is the output of our proposed system 1 .

Similar to Eq. (2), we can also estimate the DOA of the interfering speaker as $\hat{\jmath}_{T}$ to be found within the region of the customer's side.

$$
\hat{\jmath}_{T}=\underset{i_{\min }<i<0}{\operatorname{argmax}}\left(\bar{\varphi}_{T}(i)\right) .
$$

This will be used for the proposed CSP weighting in Sect. 3.5 .

\subsection{CSP Problem and Solution}

When the interfering speaker (the customer) speaks, the CSP coefficient $\bar{\varphi}_{T}\left(\hat{\imath}_{T}\right)$ is expected to be non-positive. However, as shown in Fig. 4, the original CSP-target often takes values above zero even in the regions where only the customer is speaking. Therefore, we cannot block the customer's speech in the output. We analyzed this problem and found this is caused by spatial aliasing [20].

Figure 5 shows an example of a CSP distribution affected by spatial aliasing. When the customer speaks, the main peak is observed at the index pointing to the customer. However, on the agent side, a sub-peak may be observed at an index value pointing to the agent.

Referring to Eq. (1), the CSP coefficient can be interpreted as the grand sum of each bin's contribution. 


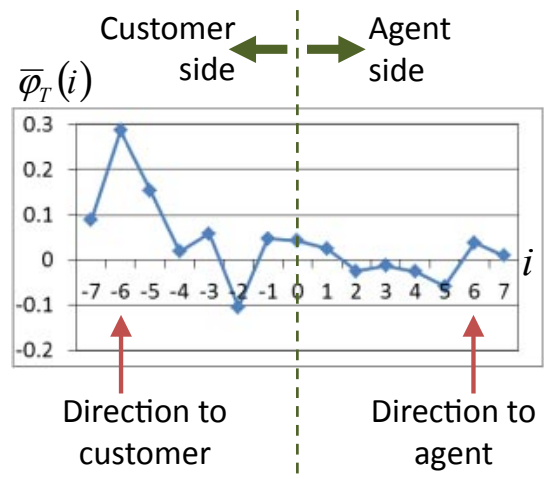

Fig. 5 Example of CSP coefficients when a customer speaks.

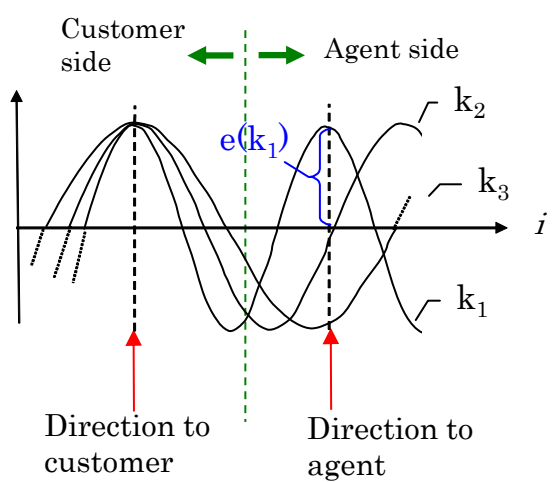

Fig. 6 Breakdown of CSP into frequency bins.

Figure 6 shows a conceptual breakdown of some components. Based on the bin number (or frequency), the component generates a fake peak at the index of the agent by spatial aliasing. For example, in Fig. 6 , the sound wave $\mathrm{k} 1$ coming from the customer's direction is observed similarly to the one from the agent's direction. Thus, it leads $\bar{\varphi}_{T}\left(\hat{\imath}_{T}\right)$ to be positive.

Therefore, we can lower the contributions of such frequency bins in order to have more suppression. If the directions both to the agent and to the customer are available, we can estimate the extent of the spatial aliasing for the bin $k$ as

$$
e_{T}(k)=\cos \left(\frac{2 \pi \cdot k \cdot\left(\hat{\imath}_{T}-\hat{\jmath}_{T}\right)}{M}\right) .
$$

$M$ is the DFT size.

Higher $e_{T}(k)$ means it is a problematic bin. We can use the lower $W_{T}(k)$ for the higher $e_{T}(k)$ as

$$
W_{T}(k)=\min \left(1.0, \quad 1.0-e_{T}(k)\right) .
$$

This is used in Eq. (1) for the weighted-CSP.

Figure 7 shows the example of $W_{T}(k)$. In this way, we can lower the contribution from the bins that have peaks at both indices pointing to the agent and the customer.

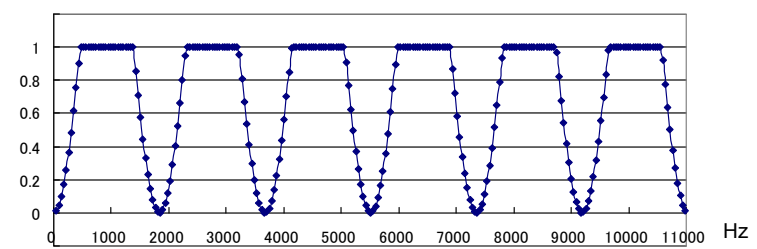

Fig. 7 Example of bin weights for CSP analysis.

Figure 4 shows the improvement by this approach. In the weighted-CSP case, CSP-target takes non-positive values whenever the customer speaks, as desired.

\subsection{Partial-binary-masking}

Binary-masking works well in a particular configuration that the two sound sources have largely separated DOAs.

DOA angle $\theta_{T}(k)$ for the bin $k$ can be determined as a correlation of the two-microphone observation with the moving average around the current frame $T$.

$$
\begin{aligned}
& R_{T}(k)=S_{1, T}(k) \cdot S_{2, T}(k)^{*} . \\
& \overline{R_{T}}(k)=\frac{1}{(2 H+1)} \sum_{h=-H}^{H} R_{T+h}(k) . \\
& \theta_{T}(k)=\angle \overline{R_{T}}(k) .
\end{aligned}
$$

Binary-masking suppresses the output of the bin whose DOA angle is not in the target area. When spatial aliasing occurs, this judgment goes wrong. This paper introduced the upper-limit frequency so to avoid binary-masking to be used in the region affected by spatial aliasing. Here is our partial-binary-mask $B_{T}(k)$.

$$
\begin{array}{ll}
B_{T}(k)=1 & \left(k>k_{\text {limit }}\right) \\
B_{T}(k)=1 & \left(k \leq k_{\text {limit }}, \theta_{T}(k)>0\right) \\
B_{T}(k)=0 & \text { (otherwise }) .
\end{array}
$$

We do not mask if the bin number is higher than the limiting bin $k_{\text {limit }}$, which is given by

$$
k_{\text {limit }}=\gamma \cdot(M \cdot c) /(2 \cdot d \cdot f) .
$$

Here, $\gamma$ is a safety margin less than 1.0. We set it to 0.75 in our experiments. So, in our configuration, the upper frequency is around $1 \mathrm{kHz}$. Although it does not fully mask the unwanted speech, it can sufficiently mask the first formant part of vowels.

This binary-mask is applied for the output of the postfiltering.

$$
V_{T}(k)=B_{T}(k) \cdot Y_{T}(k) .
$$

Finally, we have the output of our proposed system 2

$$
Z_{T}(k)=\max \left(V_{T}(k), \quad \beta \cdot N(k)\right) .
$$


NRR (dB)

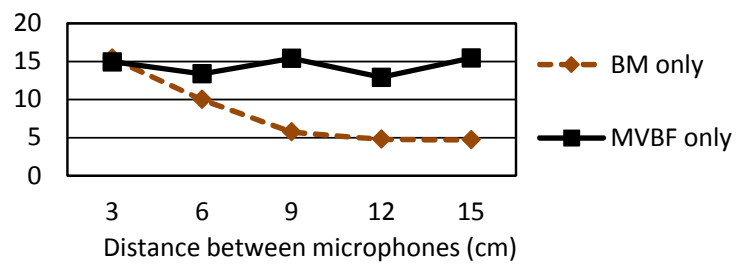

Fig. 8 Noise Reduction Rate in $\mathrm{dB}$ depending on the distance between microphones. BM indicates partialbinary-masking.

\section{PRELIMINARY EXPERIMENTS}

In this section, we will find a suitable distance between microphones to maximize the performance of binarymasking and adaptive beamformer (MVBF).

\subsection{Experimental Setup}

In a sound proof chamber, two omnidirectional microphones (Sony ECM-55B) were placed along the line pointing to the two loud speakers. The distance between the microphones was varied from $3 \mathrm{~cm}$ to $15 \mathrm{~cm}$. The distance between the loud speakers was $70 \mathrm{~cm}$. One loud speaker is for a male subject speaker and the other is for a female speaker to play back their utterances alternately and simultaneously.

\subsection{Noise Suppression Capability}

Noise Reduction Rate (NRR) measures how much sound power is reduced in non-speech segments while the interfering speaker is speaking. Figure 8 shows that partialbinary-masking suppressed the unwanted speech more with narrower microphone array such as $3 \mathrm{~cm}$ spacing. On the other hand, MVBF performed almost equally in NRR for various microphone spacing.

\subsection{Speech Separation Capability}

NRR in Sect. 4.2 is not sufficient to assure the higher ASR performance, because it does not measure distortion in the speech segment. For the mixed speech segment, we used cepstrum distance to measure how well the contaminated speech was restored. Figure 9 shows that the cepstrum distance with partial-binary-masking was generally higher than with MVBF. It means partial-binarymasking involves more speech distortion in the speech separation. MVBF separated the mixed speech better with wider microphone spacing.

Considering the above evaluation results on NRR and cepstrum distance, we decided to use $12 \mathrm{~cm}$ for the distance between microphones. As Fig. 9 suggests, we could expect better results using a slightly wider distance, but it also increases the risk of spatial aliasing.

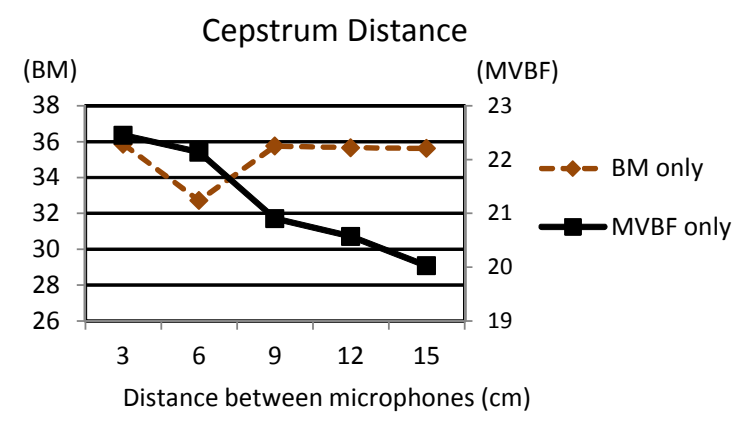

Fig. 9 Cepstrum distance between the reference speech and the separated mixed speech. Cepstrum distance was calculated without zero-th cepstrum $(\mathrm{c} 0)$.

\section{EXPERIMENTS}

\subsection{Experimental Setup}

For this paper, an evaluation was performed for a Type 1 in Fig. 1. In a small, quiet meeting room whose size is approximately $3.2 \mathrm{~m}$ by $3.2 \mathrm{~m}$, two omnidirectional microphones (Sony ECM-55B) were placed on a table between two subject speakers acting as an agent and a customer. The two subject speakers alternately read hundred sentences for each. The distance between the microphones was $12 \mathrm{~cm}$. The distance from the subject speaker to the microphone was ranged roughly from $50 \mathrm{~cm}$ to $80 \mathrm{~cm}$. The signal powers to the ambient noise power were about $29.3 \mathrm{~dB}$ for the agent and about $26.8 \mathrm{~dB}$ for the customer, as measured with the near-end microphones. The beamformer operated at $22.05 \mathrm{kHz}$ sampling frequency. For the experiment, we had two output streams both for the agent and the customer. The performance of the speech separation and suppression can be measured by the error rate of ASR. We can detect a leak sound from the interfering speaker, by increased insertion errors of ASR.

Because the ASR decoder accepts segmented PCM data sampled at $16 \mathrm{kHz}$, the beamformer output was downsampled and segmented. The segmentation was performed so to split at the middle of the non-speech segment of the target speaker. Therefore, each segment included long silence before and after the speech period. Please note the silence contains interfering speech and true silence. The ASR decoder has its Voice Activity Detection (VAD) and the acoustic model was trained with data processed with SS and flooring.

The topic of the spoken sentences was a travel conversation and the matched language model was used for the decoding. Stationary noise component for SS was estimated when both subject speakers are silent.

We also tested mixed speech scenario by simulation. Using the recording of the alternate speech scenario above, a part of customer's speech segment was extracted and 
Table 1 Evaluation results for proposed system 1. Character Error Rate (CER) \% and Noise Reduction Rate (NRR) in dB. \#T is the total number of reference characters, \#S is the total number of substitution errors, \#I is the total number of insertion errors and \#D is the total number of deletion errors.

(a) Alternate speech case.

\begin{tabular}{|c|c|c|c|c|c|c|c|}
\hline \multirow{2}{*}{ System } & & \multicolumn{5}{|c|}{ ASR results } & \multirow{2}{*}{ NRR } \\
\hline & & \#T & $\# \mathrm{~S}$ & $\# \mathrm{I}$ & \#D & CER & \\
\hline Case 0) & Oracle (manually segmented alternate speech) & 2,547 & 99 & 10 & 53 & 6.4 & - \\
\hline Case 1) & Baseline (Single microphone) & 2,547 & 375 & 2,183 & 24 & 101.4 & - \\
\hline Case 2) & MVBF & 2,547 & 337 & 2,032 & 20 & 93.8 & 6.9 \\
\hline Case 3) & $\mathrm{MVBF}+\mathrm{SS}+$ Floor & 2,547 & 335 & 2,043 & 21 & 94.2 & 7.0 \\
\hline Case 4) & MVBF + SS + Zelinski_PF + Floor & 2,547 & 368 & 1,241 & 39 & 64.7 & 10.2 \\
\hline Case 5) & MVBF + SS + CSP_PF + Floor & 2,547 & 218 & 668 & 33 & 36.1 & 14.8 \\
\hline Case 6) & $\begin{array}{l}\text { MVBF }+ \text { SS }+ \text { w-CSP_PF + Floor } \\
\text { (Proposed system 1) }\end{array}$ & 2,547 & 87 & 20 & 44 & 5.9 & 16.4 \\
\hline Case 7) & $\mathrm{MVBF}+\mathrm{SS}+$ Floor + w-CSP_PF & 2,547 & 121 & 77 & 51 & 9.8 & $\mathbf{1 7 . 4}$ \\
\hline
\end{tabular}

(b) Mixed speech case.

\begin{tabular}{|c|c|c|c|c|c|c|c|}
\hline \multirow{2}{*}{ System } & & \multicolumn{5}{|c|}{ ASR results } & \multirow{2}{*}{ NRR } \\
\hline & & $\# \mathrm{~T}$ & $\# \mathrm{~S}$ & \#I & \#D & CER & \\
\hline Case 0) & Oracle (manually segmented alternate speech) & 1,263 & 44 & 6 & 16 & 5.2 & - \\
\hline Case 1) & Baseline (Single microphone) & 1,263 & 444 & 1,934 & 9 & 189.0 & - \\
\hline Case 2) & MVBF & 1,263 & 235 & 1,733 & 8 & 156.5 & 6.3 \\
\hline Case 3) & MVBF + SS + Floor & 1,263 & 233 & 1,747 & 8 & 157.4 & 6.5 \\
\hline Case 4) & MVBF + SS + Zelinski_PF + Floor & 1,263 & 260 & 1,011 & 6 & 101.1 & 8.7 \\
\hline Case 5) & MVBF + SS + CSP_PF + Floor & 1,263 & 240 & 1,155 & 6 & 111.0 & 11.9 \\
\hline Case 6) & $\begin{array}{l}\text { MVBF + SS + w-CSP_PF + Floor } \\
\text { (Proposed system 1) }\end{array}$ & 1,263 & 86 & 24 & 32 & 11.2 & 15.5 \\
\hline Case 7) & $\mathrm{MVBF}+\mathrm{SS}+$ Floor + w-CSP_PF & 1,263 & 112 & 91 & 31 & 18.5 & 15.9 \\
\hline
\end{tabular}

scaled by $50 \%$, and then they were added over the entire recording of the alternate speech in the stereo format. This was a very challenging task, because the agent speech was mixed with the customer's speech. There was almost no complete silence in the observation. ASR was performed only for the agent speech consisting of one hundred utterances.

\subsection{Noise Reduction Rate}

For the evaluation metric, we also used Noise Reduction Rate. In order to make it scale-invariant, we estimated it from Signal-to-Noise Ratio (SNR) in dB as

$$
N R R=S N R_{\text {current }}-S N R_{\text {baseline }} .
$$

This uses an assumption that the speech part is identical between the current (processed) data and the baseline data, except scaling. This is not always true especially for mixed speech cases, because MVBF reduces the power in the speech segments. However, we accept it for an approximation. Therefore, it should be noted that NRR in this paper may indicate smaller values than the actual.

\subsection{Experimental Results}

Table 1 shows the experimental results without binary masking for the evaluation of our proposed system 1 .
Case 0 is for the reference. The alternate speech data was manually segmented so to have the target speech only. This result can be considered as an upper bound of the performance. Case 1 is the baseline using single microphone nearest to the speaker. The error rate was so high because it includes the interfering speech without any reduction. Case 2 is the simple MVBF system. It did a lot for the mixed speech, but did a little for the alternate speech. MVBF did some speech separation in the mixed speech segments, but it did not sufficiently suppress the output when only the interfering speaker talks. The leak sound from the interfering speaker increased insertion errors in ASR. In Case 3, the combination with SS did not make much difference, because the ambient noise was small in this recording. Case 4 introduced Zelinski's postfilter (Zelinski_PF) to Case 3. It further reduced the errors in favor of the suppression capability. But it is still not sufficient. Case 5 replaced Zelinski_PF with CSPbased post-filter (CSP_PF). CSP_PF worked better than Zelinski_PF in the alternate speech case. This is because Zelinski_PF is not designed for a directional noise and it had remaining noise at several frequency bands corresponding to the spatial aliasing. On the other hand, Zelinski_PF was better for the mixed speech case, because it works for each frequency bin to split overlapped speech. 
Table 2 Evaluation results for proposed system 2. Character Error Rate (CER) \% and Noise Reduction Rate (NRR) in dB. $\mathrm{BM}$ indicates partial-binary-masking. The definition of \#T, \#S, \#I and \#D are the same as Table 1.

(a) Alternate speech case.

\begin{tabular}{|c|c|c|c|c|c|c|c|}
\hline \multirow{2}{*}{ System } & & \multicolumn{5}{|c|}{ ASR results } & \multirow{2}{*}{ NRR } \\
\hline & & $\# \mathrm{~T}$ & $\# \mathrm{~S}$ & $\# \mathrm{I}$ & \#D & CER & \\
\hline Case 0) & Oracle (manually segmented alternate speech) & 2,547 & 99 & 10 & 53 & 6.4 & - \\
\hline Case 1) & Baseline (Single microphone) & 2,547 & 375 & 2,183 & 24 & 101.4 & - \\
\hline Case 8) & $\mathrm{MVBF}+\mathrm{BM}$ & 2,547 & 565 & 788 & 50 & 55.1 & 13.0 \\
\hline Case 9) & $\mathrm{MVBF}+\mathrm{SS}+\mathrm{BM}+$ Floor & 2,547 & 407 & 933 & 38 & 54.1 & 12.9 \\
\hline Case 10) & MVBF + SS + CSP_PF + BM + Floor & 2,547 & 155 & 158 & 47 & 14.1 & 16.3 \\
\hline Case 11) & $\begin{array}{l}\text { MVBF }+ \text { SS }+ \text { w-CSP_PF }+ \text { BM }+ \text { Floor } \\
\text { (Proposed system 2) }\end{array}$ & 2,547 & 96 & 11 & 44 & 5.9 & 16.5 \\
\hline \multicolumn{8}{|c|}{ (b) Mixed speech case. } \\
\hline \multirow{2}{*}{ System } & & \multicolumn{5}{|c|}{ ASR results } & \multirow{2}{*}{ NRR } \\
\hline & & $\# \mathrm{~T}$ & $\# \mathrm{~S}$ & $\# \mathrm{I}$ & \#D & CER & \\
\hline Case 0) & Oracle (manually segmented alternate speech) & 1,263 & 44 & 6 & 16 & 5.2 & - \\
\hline Case 1) & Baseline (Single microphone) & 1,263 & 444 & 1,934 & 9 & 189.0 & - \\
\hline Case 8) & $\mathrm{MVBF}+\mathrm{BM}$ & 1,263 & 450 & 714 & 11 & 93.0 & 14.0 \\
\hline Case 9) & $\mathrm{MVBF}+\mathrm{SS}+\mathrm{BM}+$ Floor & 1,263 & 326 & 795 & 5 & 89.2 & 13.8 \\
\hline Case 10) & $\mathrm{MVBF}+\mathrm{SS}+$ CSP_PF + BM + Floor & 1,263 & 177 & 204 & 21 & 31.8 & 15.8 \\
\hline Case 11) & $\begin{array}{l}\text { MVBF }+ \text { SS }+ \text { w-CSP_PF }+ \text { BM }+ \text { Floor } \\
\text { (Proposed system 2) }\end{array}$ & 1,263 & 52 & 6 & 18 & 6.0 & 16.4 \\
\hline
\end{tabular}

Case 6 is our proposed system 1 including w-CSP_PF. Comparing Case 6 with Case 5, w-CSP_PF decreased the error rate drastically from CSP PF. The improvement was mostly gained by the less insertion errors. Also, it showed higher NRR. For another finding, Case 6 had less substitution errors than the oracle results (Case 0) in the alternate speech case. This is the benefit of MVBF while Case 0 uses only a single channel information. Case 7 is for the reference. It performed the flooring just after spectral subtraction (SS). This sequence order is more natural for SS, but the accuracy was not optimum. On the other hand, nominal NRR was higher than Case 6, because the floored output was also attenuated. Although the SS did not have significant factor in this test-set, the flooring played a substantial role for ASR accuracy if it was placed after the post-filter, as we proposed.

As the result, our proposed system 1 (Case 6) successfully outperformed the oracle results in the alternate speech case. In the mixed speech case, our proposed system 1 also showed fairly nice improvement. although it did not reach the oracle case performance.

Table 2 shows the experimental results combined with partial-binary-masking for the evaluation of our proposed system 2. It shares the oracle and baseline results (Case 0 and Case 1) with Table 1.

Case 8 corresponds to Case 2, Case 9 corresponds to Case 3 and Case 10 corresponds to Case 5. They showed addition of the partial-binary-masking generally helped the ASR accuracy in favor of the reduced insertion errors.
In the alternate speech case, our proposed system 2 (Case 11) showed the error rate almost same as Case 6. In the details, the insertion errors were less and the substitution errors were more. This implies binary-masking approach could involve some speech distortion in the trade off of noise reduction. In the mixed speech case, the benefit of partial-binary-masking was much distinct. When it was combined with our proposed post-filter (w-CSP_PF), our proposed system 2 (Case 11) reduced the errors by $96.8 \%$ from the baseline, and it was close to the oracle result. This is a remarkable result considering this is a mixed speech condition.

\section{CONCLUDING REMARKS}

This paper introduced a small-scale microphone array system specially designed for use at a teller's counter. It uses two microphones installed on the line between the speakers. Our objective is to transcribe only the agent's speech by ASR. We found the post-filtering using the standard CSP often becomes inaccurate due to the spatial aliasing with an interfering speaker. We demonstrated that this problem can be mitigated by introducing a weighted CSP approach that lowers the contribution from the bins affected by the aliasing. We also demonstrated the merit of the flooring placed after the post-filtering, so that the residual speech can be less audible. The proposed method was further combined with partial-binary-masking and it achieved practical performance both in the alternate speech case and in the mixed speech case. 


\section{ACKNOWLEDGEMENT}

We would like to express my special thanks to Prof. Masafumi Nishimura who provided valuable comments to help this research succeed.

\section{REFERENCES}

[1] S. Araki, H. Sawada, R. Mukai and S. Makino, "A novel blind source separation method with observation vector clustering," Proc. IWAENC, pp. 117-120 (2005).

[2] M. Aoki, M. Okamoto, S. Aoki, H. Matsui, T. Sakurai and Y. Kaneda, "Sound source segregation based on estimating incident angle of each frequency component of input signals acquired by multiple microphones," Acoust. Sci. \& Tech., 22, 149-157 (2001).

[3] Y. Mori, H. Saruwatari, T. Takatani, S. Ukai, K. Shikano, T. Hiekata and T. Morita, "Real-time implementation of twostage blind source separation combining SIMO-ICA and binary masking," Proc. IWAENC, pp. 229-232 (2005).

[4] F. Asano, H. Asoh and T. Matsui, "Sound source localization and separation in near field," IEICE Trans., E83-A, 2286-2294 (2000).

[5] L. J. Griffiths and C. W. Jim, "An alternative approach to linearly constrained adaptive beamforming," IEEE Trans. Antennas Propag., AP-30, 27-34 (1982).

[6] Y. Ohashi, T. Nishikawa, H. Saruwatari, A. Lee and K. Shikano, "Noise-robust hands-free speech recognition based on spatial subtraction array and known noise superimposition," Proc. Intelligent Robots and Systems, pp. 2328-2332 (2005).

[7] O. Ichikawa, T. Takiguchi and M. Nishimura, "Speech enhancement by profile fitting method," IEICE Trans. Inf. Syst., E86-D, 514-521 (2003).

[8] P. Smaragdis and J. C. Brown, "Non-negative matrix factorization for polyphonic music transcription," Proc. WASPAA, pp. 177-180 (2003).

[9] A. Ozerov, C. Févotte, R. Blouet and J. L. Durrieu, "Multichannel nonnegative tensor factorization with structured constraints for user-guided audio source separation," Proc. ICASSP 2011, pp. 257-260 (2011).

[10] R. Zielinski, "A microphone array with adaptive post-filtering for noise reduction in reverberant rooms," Proc. ICASSP 88, pp. 2578-2581 (1988).

[11] I. A. McCowan, C. Marro and L. Mauuary, "Robust speech recognition using near-field superdirective beamforming with post-filtering," Proc. ICASSP 2000, Vol. 3, pp. 1723-1726 (2000).

[12] C. Marro, Y. Mahieux and K. U. Simmer, "Analysis of noise reduction and dereverberation techiniques based on microphone arrays with postfiltering," IEEE Trans. Speech Audio Process., 6, 240-259 (1998).

[13] N. Ito, N. Ono and S. Sagayama, "A blind noise decorrelation approach with crystal arrays on designing post-filters for diffuse noise suppression," Proc. ICASSP 2008, pp. 317-320 (2008).

[14] Y. Nagata, T. Fujioka and M. Abe, "Speech enhancement based on auto gain control," IEEE Trans. Audio Speech Lang. Process., 14, 177-190 (2006).

[15] M. Omologo and P. Svaizer, "Acoustic event localization using a crosspower-spectrum phase based technique," Proc. ICASSP 94, pp. 273-276 (1994).

[16] Y. Denda, T. Nishiura and Y. Yamashita, "Robust talker direction estimation based on weighted CSP analysis and maximum likelihood estimation," IEICE Trans. Inf. Syst., E89D, 1050-1057 (2006).

[17] O. Ichikawa, T. Fukuda and M. Nishimura, "DOA estimation with local-peak-weighted CSP," EURASIP J. Adv. Signal Process., 2010, Article No. 3 (2010).

[18] T. Fukuda, O. Ichikawa and M. Nishimura, Japan Patent No. 5339501 (2008).

[19] T. Fukuda, O. Ichikawa and M. Nishimura, Japan Patent No. 5156260 (2007).

[20] O. Ichikawa, T. Fukuda and M. Nishimura, Japan Patent No. 4906908 (2009).

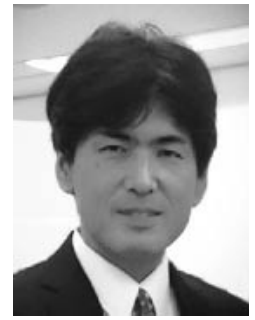

Osamu Ichikawa is a research staff member at the IBM Research - Tokyo and belongs to the speech technology group. He received B.E. and M.E. degrees in Aeronautical Engineering from Tokyo University, Japan, in 1986 and 1988, respectively, and a Ph.D. degree in Information Science from Nara Institute of Science and Technology, Japan, in 2008.

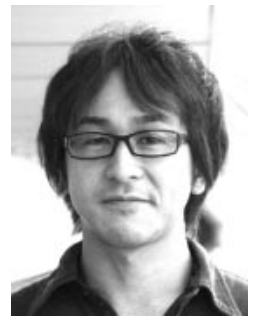

Takashi Fukuda received his Ph.D. degree from Toyohashi University of Technology, Japan, in 2005. He has been with the speech technology group, IBM Research - Tokyo since 2005. Dr. Fukuda received the 28th Awaya Kiyoshi Young Researcher Award in 2010, IEICE ISS Young Researcher's Award in Speech Field in 2012, and IPSJ Yamashita SIG Research Award in 2013.

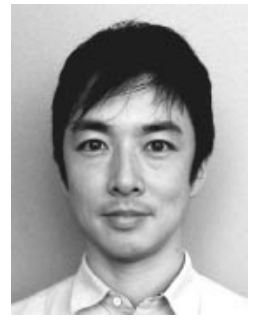

Ryuki Tachibana is the manager of the Speech Technology group at IBM Research Tokyo. His expertise and research interests are in the area of speech synthesis, speech recognition, and audio watermarking. He received his B.E. and M.E. in aerospace engineering from the University of Tokyo, Japan, in 1996 and 1998, and his Dr. Eng. degree from Osaka University in 2007. 